\title{
Use of Fourier Transform Infrared Spectroscopy and Chemometric Data Analysis To Evaluate Damage and Age in Mushrooms (Agaricus bisporus) Grown in Ireland
}

\author{
Aoife O'Gorman \\ Technological University Dublin, aoife.ogorman@tudublin.ie \\ Gerard Downey \\ Teagasc \\ Aoife Gowen \\ University College Dublin, aoife.gowen@ucd.ie
}

See next page for additional authors

Follow this and additional works at: https://arrow.tudublin.ie/schfsehart

Part of the Food Chemistry Commons

\section{Recommended Citation \\ O'Gorman, A. (2010): Use of Fourier Transform Infrared Spectroscopy and Chemometric Data Analysis To Evaluate Damage and Age in Mushrooms (Agaricus bisporus) Grown in Ireland. Journal of Agriculture and Food Chemistry, 58 (13), pp.7770-7776. doi:10.1021/jf101123a}

This Article is brought to you for free and open access by the School of Food Science and Environmental Health at ARROW@TU Dublin. It has been accepted for inclusion in Articles by an authorized administrator of ARROW@TU

Dublin. For more information, please contact

arrow.admin@tudublin.ie, aisling.coyne@tudublin.ie, gerard.connolly@tudublin.ie.

Funder: Irish Department of Agriculture and Food under the Food Institutional Research Measure (FIRM),

supported through EU and national funds.

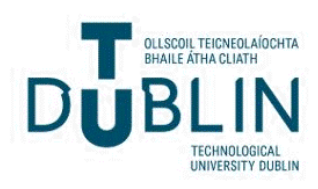




\section{Authors}

Aoife O'Gorman, Gerard Downey, Aoife Gowen, Catherine Barry-Ryan, and Jesus Maria Frias

This article is available at ARROW@TU Dublin: https://arrow.tudublin.ie/schfsehart/68 
Use of Fourier-transform infrared spectroscopy and chemometric data analysis to evaluate damage and age in mushrooms (Agaricus bisporus) grown in Ireland

Aoife O'Gorman ${ }^{a}$, Gerard Downey ${ }^{b}$, Aoife A. Gowen ${ }^{c}$, Catherine Barry-Ryan ${ }^{a}$, and Jesus M. Frias ${ }^{\mathrm{a}}$

${ }^{\text {a }}$ School of Food Science \& Environmental Health, Dublin Institute of Technology, Cathal Brugha Street, Dublin 1, Ireland

${ }^{\mathrm{b}}$ Teagasc, Ashtown Food Research Centre, Ashtown, Dublin 15, Ireland

${ }^{c}$ Biosystems Engineering, School of Agriculture, Food Science and Veterinary

Medicine, University College Dublin, Dublin 4, Ireland

* Corresponding author. Tel +3531402 4459, Fax +35314024495

E-mail: jesus.frias@dit.ie 


\section{Abstract}

4 The aim of this research was to investigate whether the chemical changes induced by

5 mechanical damage and aging of mushrooms can be (a) detected in the mid-infrared

6 absorption region and (b) identified using chemometric data analysis. Mushrooms

7 grown under controlled conditions were bruise-damaged by vibration to simulate

8 damage during normal transportation. Damaged and non-damaged mushrooms were

9 stored for up to 7 days post-harvest. Principal component analysis of FTIR spectra

10 showed evidence that physical damage had an effect on tissue structure and the aging

11 process. Random forest classification models were used to predict damage in 12 mushrooms producing models with error rates of 5.9 and $9.8 \%$ with specific 13 wavenumbers identified as important variables for identifying damage, PLS models 14 were developed producing models with low levels of misclassification. Modeling 15 post-harvest age in mushrooms using random forests and PLS resulted with high error 16 rates and misclassification; however, random forest models had the ability to correctly 17 classify $82 \%$ of day zero samples, which may be a useful tool in discriminating 18 between 'fresh' and old mushrooms. This study highlights the usefulness of FTIR 19 spectroscopy coupled with chemometric data analysis in particular for evaluating 20 damage in mushrooms and with the possibility of developing a monitoring system for 21 damaged mushrooms using the FTIR 'fingerprint' region.

25 Key words: FTIR spectroscopy; chemometrics; mushrooms; aging; damage 
Mushroom cultivation is a worldwide business with the global market valued at over $\$ 45$ billion in 2005 (1). In Ireland more than 60, 000 tons of button mushrooms (Agaricus bisporus) are produced annually, making them one of the most important horticultural crops grown (2). Mushrooms are one of the most perishable food products with a maximum shelf-life of 3-4 days at ambient temperature (3) mainly because they have no cuticle to offer protection from physical damage, microbial attack or water loss (4). They may be bruised easily by physical stress during harvesting, handling and transportation. This mechanical damage triggers a browning

41 process which is the major cause of loss of value in the market $(5,6)$. A second significant factor determining mushroom quality is time elapsed between harvesting and delivery to the marketplace. Post-harvest age is particularly important for any mushroom exporting country (i.e. Ireland) for which access to the food markets in larger, neighboring countries within Europe is vital. There is a need for a method which would allow objective evaluation of mushroom quality to ensure that only high quality produce reaches the retail market and that is able to produce information on the metabolites in mushrooms affected by senescence and damage (7). the rapid, reagentless and high-throughput analysis of a diverse range of samples (8).

51 Its importance lies in its ability to allow rapid and simultaneous characterization of 52 different functional groups such as lipids, proteins, nucleic acids and polysaccharides 53 (9-12) in biological molecules and complex structures. FTIR spectroscopy is an 54 important tool used for quality control and process monitoring in the food industry 55 because it is less expensive, has better performance and is easier to use than other 
methods (13). In the same way, FTIR spectroscopy has been used as a fingerprinting tool to study response of cells to various stressing situations (14-16).

A key to the successful operation of this technique is the availability of mathematical tools for the interrogation and mining of large spectral data sets. Principal component analysis (PCA), partial least squares (PLS) regression and random forests (RF) are chemometric tools that have been successfully used to extract information from FTIR data $(17,18)$.

The objective of this study was to investigate the damage and aging of mushrooms grown in Ireland using FTIR spectroscopy in order to (a) differentiate between damaged and undamaged mushrooms and (b) to determine mushroom post-harvest age. The ability to develop a tool that could detect physical damage before browning becomes visible would be of importance to the mushroom industry and could reduce economic losses.

\section{MATERIALS AND METHODS}

Mushrooms. Second flush mushrooms were grown at the Teagasc Research Centre Kinsealy (Dublin, Ireland), harvested damage-free. A set of 160 closed cap, defect-free Agaricus bisporus strain Sylvan A15 (Sylvan Spawn Ltd., Peterborough, United Kingdom) mushrooms (3-5 cm cap diameter) were selected for this study and immediately transported by road to the testing laboratory. Special trays were designed to hold mushrooms by the stem using a metal grid to avoid contact between (a) mushrooms and (b) between the top of mushroom caps and the tray lid during transportation. Mushrooms arrived at the laboratory premises within $1 \mathrm{~h}$ after harvesting and where either damaged for the specified time length or remained damage free and then stored at $4^{\circ} \mathrm{C}$ until required for analysis.

1 Mushroom treatments. Mushrooms $(n=160)$ were harvested in the conventional 
manner on a single occasion. On the day of harvest, a subset $(n=80)$ was subjected to physical damage using a mechanical shaker (Gyrotory G2, New Brunswick Scientific Co. USA) set at $300 \mathrm{rpm}$ (rotations per minute) for 20 minutes; these samples were labeled as damaged (D). The remaining 80 mushrooms were untreated and labeled undamaged (UD). Ten (10) damaged and 10 undamaged mushrooms were selected at random from their respective sub-sets on the day of harvesting and prepared for spectroscopic analysis (see below); these are referred to as day 0 samples. The remainder of the mushrooms (70 each of damaged and undamaged) were placed in plastic punnets (six mushrooms per punnet) and stored as separate batches at $4^{\circ} \mathrm{C}$ in a controlled temperature facility. On each of 7 consecutive days of such storage, a set of 10 damaged and 10 undamaged mushrooms was randomly selected, removed from storage and prepared for FTIR analysis.

FTIR spectroscopy. Sample preparation involved the manual dissection of each mushroom into its three main tissue types (cap, gills and stalk) before freezing overnight at $-70^{\circ} \mathrm{C}$ in a cryogenic refrigerator (Polar $340 \mathrm{~V}$ : Angelantoni Industrie spA, Massa Martana, Italy) followed by freeze-drying (Micro-modulyo, EC Apparatus Inc, New York, USA) for $24 \mathrm{~h}$. Freeze-dried samples were manually ground into fine particles using a pestle and mortar. Then, $9 \mathrm{mg}(3 \% \mathrm{w} / \mathrm{w})$ of each sample was mixed with $291 \mathrm{mg}(97 \%$ w/w) KBr (Sigma Aldrich, Dublin, Ireland). $\mathrm{KBr}$ pellets were prepared by exerting pressure of $100 \mathrm{~kg} / \mathrm{cm}^{2}(1200 \mathrm{psi})$ for approximately $2 \mathrm{~min}$ in a pellet press (Specac, UK). To eliminate any interference which might be caused by variation in pellet thickness different pellets were prepared from the same sample and their infrared spectra compared. These samples were identical with their average spectra used for analysis (19). 
107 Waltham, MA, USA) over the frequency range 4000-400 $\mathrm{cm}^{-1}$. One hundred scans of 108 each pellet were collected at $4 \mathrm{~cm}^{-1}$ resolution at room temperature using OMNIC 109 software (version ESP 5.1). The average of the 100 scans was used for further data 110 analysis. FTIR spectral data were discretized resulting in spectra containing 1868 111 individual points (discretised every $2 \mathrm{~cm}^{-1}$ ) for chemometric analysis.

112 Chemometric data analyses. Multivariate models for damage and age prediction 113 in mushrooms using both raw (i.e. unmodified) and pre-treated spectral data were 114 developed; the pre-treatment used was standard normal variate [SNV] and was 115 intended to reduce scatter-induced effects in the spectra (20). The frequency region 116 studied was 2000-400 $\mathrm{cm}^{-1}$ (fingerprint region); this spectral range encompasses 117 absorptions from most of the chemical species present and attenuation of the dataset 118 in this way avoids spectral regions which have low information content and may 119 therefore interfere with effective model development.

120 Random forest modeling achieves a classification by constructing a series of 121 decision trees (21) and takes input variables down all trees in order to optimize 122 classification. Each tree is constructed using a different bootstrap sample from the 123 original data, about one-third of the cases are left out of the bootstrap sample and are 124 not used in the construction of the k-th tree. These sets of unseen samples are called 125 out-of-bag (OOB) sets. RF makes use of these OOB sets in many ways, in particular 126 to give an unbiased estimate of the prediction error on unseen cases (22).

127 Random forest models were built to (a) discriminate between damaged and 128 undamaged mushrooms and (b) to predict mushroom ages. The number of trees fitted 129 to build the random forest was 1000 , the number of random wavenumbers tried at 130 every node of the tree was set at 500 after optimization and the random forest model 
131 trained was made using a stratified random sampling strategy of the sample spectra 132 that would take the same number of samples from each of the tissues. Principal 133 component analysis (PCA) was used to identify patterns in data in a way which 134 emphasizes differences and similarities. It is used to indicate relationships among 135 groups of variables in a data set and show relationships that might exist between 136 objects (23).

137 Partial least squares (PLS) regression was applied to the spectral data sets to 138 develop a quantitative model for prediction of the age of damaged mushrooms. A 139 common problem in development of multivariate prediction models is selection of the 140 optimum number of PLS loadings; often, this selection is based on an examination of 141 the RMSECV but identification of a minimum is not always possible or unambiguous 142 and sub-optimal models incur a significant risk of overfitting. Experience has shown 143 that this can be a problem when parameters which are of practical relevance, such as 144 post-harvest age or damage, but have unclear molecular basis are being modeled. In 145 order to avoid overfitting, model cross validation was employed as follows:

146 1. Samples were randomly-designated from each tissue/damage status/time grouping as calibration $(60 \%)$ or validation $(40 \%)$ samples. The validation

1502 2. The model optimization step was carried out in order to estimate the optimal 151 dimensionality of the PLS model built on the calibration set. The method employed for this was based on the observation that an indication of overfitting is the appearance of noise in regression vectors; this takes the form of a reduction in apparent structure and the presence of sharp peaks with a high degree of directional oscillation. A simple method (24) for objectivity 
quantifying the shape of a regression vector, combined with the root mean square error of cross-validation (RMSECV) for the calibration set was applied in this study.

159 3. The random sample designation, model development and evaluation were performed 100 times. At the end of this cycle, models were initially examined partial least squares discrimination analysis (PLS-DA). For PLS-DA, a dummy Y-

166 variable was assigned to each mushroom tissue sample, 1 for damaged and 0 for undamaged. PLS-DA calibration models were developed and assessed using 100 randomly-populated calibration and validation sample sets.

Principal component analysis (PCA) and partial least squares (PLS) regression were performed using MATLAB and The Unscrambler software (v.9.7; Camo A/S, Oslo,

171 Norway). The routine for selection of the optimum number of PLS loadings was also performed in MATLAB. Random forest modeling was performed using R 2.8.0 (25).

\section{RESULTS AND DISCUSSION}

Spectral data. Average raw spectra of each of the three tissue types collected from all the damaged and undamaged samples (day 0-7 in each case) are shown in

178 Figure 1(a, b and $\mathrm{c})$. A number of observations may be made on these spectra. 179 Firstly, the major feature is a vertical offset from one average plot to another; this 180 offset originates in light scatter effects and may be a complication in further data 181 analysis. Average spectra of the three tissue types also bear a close resemblance to 
each other; there is little visible difference in peak minima locations in Figure 1. In terms of minima locations, there are major bands at 1650,1090,1020, $935 \mathrm{~cm}^{-1}$; minor minima may be seen at 1560, 1150 and $1050 \mathrm{~cm}^{-1}$ (Figure 2). Unambiguous identification of the molecular source of features in mid-infrared spectra of biological material is difficult but the peak at 1650 may be attributed to an amide I group while at $1560 \mathrm{~cm}^{-1}$ may be identified as resulting from amide II groups $(26,27)$. Both major absorbance peaks at 1090 and $1020 \mathrm{~cm}^{-1}$ have been attributed as structures in chitin, a major structural polysaccharide in mushrooms; absorbance at $1090 \mathrm{~cm}^{-1}$ may also arise from secondary alcohols. Smaller features at 1150 and $1050 \mathrm{~cm}^{-1}$ have been attributed to tertiary and primary alcohol structures (28). Minima at 935, 890 and 874 $\mathrm{cm}^{-1}$ bands correspond to $\alpha$ - or $\beta$ - anomer $\mathrm{C}_{1}-\mathrm{H}$ deformations. The bands at 935 and $890 \mathrm{~cm}^{-1}$ are attributed to glucan bands, while the band at $874 \mathrm{~cm}^{-1}$ is assigned to a mannan band (29-31). An inability to attribute all spectral features is a common feature of spectroscopy of complex biological matrices but the presence of such spectral detail implies the detection of a significant quantity of information which may be usefully interrogated by multivariate mathematical methods.

Principal component analysis (PCA). Undamaged samples were studied separately on the basis of their tissue type i.e. caps, gills and stalks. Initial PCA of the mushroom caps data revealed a single sample (day 7) which lay anomalously at some significant distance from the others; this was deleted and the resulting score plot is shown in Figure 3 for PC1 vs PC2; these first two principal components accounted for 97 and $2 \%$ respectively of the total variance in the spectral dataset and some sample clustering on the basis of storage time is readily apparent. As a general observation, it may be stated that the majority of the day 0 mushroom caps have a score value on PC1 greater than zero and are therefore located on the right-hand-side of Figure 3a. 
207 While there are indications that samples of different storage time cluster together, the 208 spread of these clusters is quite large and it is not possible to readily discern any trend 209 relating plot position and storage time in the plots. There is a suggestion that the 210 dispersion of the samples decreases as the length of storage time increases. With 211 regard to undamaged gill tissue, observations similar to those made above in relation 212 to undamaged caps may be made although the distribution patterns are somewhat 213 different.

214 In the case of damaged mushroom tissues, a different pattern was found. It is 215 clear from Figure 3c, d and e that day 0 samples clustered together but separately 216 from those of day 1 to day 7 samples irrespective of tissue type. This strongly 217 suggested that physical damage had a significant effect on tissue structure and the 218 subsequent aging process. Some implications regarding the rate of change of 219 mushroom tissue composition with aging may be garnered from the observation that 220 separation of day 0 from all other subsequent days accounts for the most variation in 221 the spectral collection of damaged mushroom caps, gills and stalks.

Examination of PC loadings may provide information on the absorbing species 223 which are involved in separations observed on a PC scores plot; however, meaningful 224 interpretation of loadings arising from this dataset (data not shown) was not possible.

225 Detection of damage (random forests). The first random forest model 226 developed attempted to identify which wavenumbers could be used to predict damage 227 specifically. The model tried to predict damage in mushrooms using the IR spectra, a 228 variable indicating the tissue from which the spectra originated (cap, gill or stalk) and 229 the age of the mushroom (in days from 0-7) as explanatory variables. This resulted in 230 good classification between damaged and undamaged samples with an out-of-bag 231 error rate (OOB) of 5.9\%, sensitivity of $93.3 \%$ and specificity of $95 \%$. 
In random forests there are two measures of importance to indicate how

233 informative a particular variable (a wavenumber in our case) is, the mean decrease in 234 accuracy and the Gini index. The decrease in Gini index is not as reliable as the 235 marginal decrease in accuracy $(32,33)$ and for that reason the latter was analysed. 236 The variables containing the most importance for predicting damage in the model are 237 shown in Figure 4a. The most important variable for predicting damage was the age 238 of the mushrooms followed by the wavenumbers 1868,1870 and $1845 \mathrm{~cm}^{-1}$.

239 Induced damage in mushrooms leads to an enzymic response which is followed by 240 brown discoloration. The enzymes involved in this response, tyrosinase, or 241 polyphenol oxidases, catalyse the oxidation of phenols, which in turn promote the 242 formation of melanin like compounds. This reaction is found not only in damaged 243 mushrooms, but is also part of the natural aging process, with color in mushrooms 244 becoming darker and less firm during storage (34). The three wavenumbers identified 245 have the ability to differentiate between the chemical changes that are induced by the 246 mechanical damage and are independent of those that take place due solely to aging. 247 The three wavenumbers identified above are unassigned peaks.

248 By removing the variable age from the model a second model was built which 249 would take IR spectra of mushrooms (independently of their age) and try to predict 250 whether there is damage or not. This random forest could be used as a classifier of 251 mushroom damage and gave a very good prediction model with an OOB error rate of $2529.8 \%$, sensitivity of $89.2 \%$ and specificity of $91.2 \%$. Even receiving mushrooms 253 whose storage time after harvest was unknown the model would still classify damaged 254 and undamaged mushroom samples with a very good classification rate. The 255 variables of importance involved in this classification model are shown in Figure 4b. 
256 The most important variable for predicting damage according to the mean decrease 257 accuracy plot is tissue used in the analysis followed by the wavenumbers 1868,1870 258 and $1560 \mathrm{~cm}^{-1}$. The peak at $1560 \mathrm{~cm}^{-1}$ is attributed to amide II vibrations of proteins 259 (29). Amide II bands along with amide I bands are major regions of the protein 260 infrared spectrum. Amide II bands are associated with an out-of-phase combination 261 of in-plane C-N stretching and N-H bending of amide groups (35). Absorption of this 262 band was found to be higher in damaged samples and therefore an important variable 263 for detecting damage in mushroom samples. The wavenumbers 1868 and $1870 \mathrm{~cm}^{-1}$ 264 are unassigned.

Detection of damage (PLS). PLS-DA models were developed to discriminate 266 between undamaged and damaged mushrooms of all tissue types separately. A 267 summary of the average and dispersion of the results obtained on a percentage basis 268 for each tissue is shown in Table 2; it is apparent that misclassification errors 269 associated with all models were low, especially so in the case of gills and stalks. In 270 terms of numbers of samples misclassified, these percentages translate to 1 or 2 only 271 in each case. These results indicate that FTIR of freeze-dried mushroom tissues 272 (especially gills and stalks) may be used to discriminate between damaged and 273 undamaged mushrooms aged post-harvest from 0 to 7 days with almost complete 274 confidence.

275 Modeling damage in mushrooms has been reported in literature in 2008 by Gowen 276 and colleagues and in 2009 by Esquerre et al. (36, 37). Gowen and colleagues 277 investigated the use of hyperspectral imaging and principal components analysis 278 (PCA) to develop models to predict damage on mushroom caps with correct 279 classification ranging from 79-100\%. Using near infra-red spectroscopy and partial 280 least squares (PLS) regression, Esquerre and colleagues were able to correctly classify 
281 undamaged mushrooms from damaged ones with an overall correct classification 282 model with 99\% accuracy. The models for predicting damage using FTIR and 283 random forests correctly classified 94 and $90 \%$ of samples respectively, whilst the 284 PLS predictive models correctly classified 92-99\% of undamaged samples from 285 damaged ones. These results highlight the usefulness of FTIR and chemometrics for 286 detecting physical damage in mushrooms with the possibility of developing a 287 classification system for the industry.

288 Predicting post-harvest age (random forests). Initial random forest models were 289 built to try and predict the mushroom age from day zero to day seven (0-7) using the 290 IR spectra from the tissues and knowing whether they had been subjected to damage 291 or not with the aim to identify specific wavenumbers associated with aging. The 292 random forest model produced an OOB error rate of $32 \%$ i.e. $68 \%$ of samples were 293 correctly classified. The results of the model fit are shown in Table 3. 294 Misclassification of samples was seen for all mushroom ages particularly days 4, 5 295 and 7. Classification of day zero samples performed quite well in the model with $29682 \%$ of samples correctly classified, which leads to the possibility of using IR 297 spectroscopy as a tool to discriminate fresh mushrooms (D0) from mushrooms that 298 have been subjected to refrigeration. This type of tool could enable packers and 299 producers to avoid fraud and 'recycling' of product, supporting the evidence from 300 visual inspection. The variables of importance identified by the mean decrease 301 accuracy plot were damage, tissue type and the wavenumbers 399,952 and $1508 \mathrm{~cm}^{-1}$. 302 A second model was developed to predict age using the same approach as above but 303 removing the damage variable from the model. The model performed much the same 304 as above with an OOB error rate of 33\%; again misclassification within all sample 305 ages was seen. The model correctly classified $79 \%$ of day zero models. The 
important variables identified to predict age were tissue type and the wavenumbers 399, 952 and $1508 \mathrm{~cm}^{-1}$. The peak at $952 \mathrm{~cm}^{-1}$ is a glucan band ( $(3$-anomer $\mathrm{C}-\mathrm{H}$ deformation) (29), glucans play many different roles in the physiology of fungi, some accumulate in the cytoplasm as storage, however most are present in the cell wall

310 structure (38). This suggests that the ability to model aging in mushrooms may 311 depend on the affect of glucan levels changing in the cell wall due to natural senescence. The wavenumbers at 399 and $1508 \mathrm{~cm}^{-1}$ are unassigned. The OOB 313 errors produced to model aging were quite large $>33 \%$ which may be due to the low 314 sample numbers.

315 Predicting post-harvest age (PLS). PLS regression was applied separately to the 316 caps, gills and stalks datasets in an attempt to develop separate quantitative models for 317 prediction of the age of mushrooms, both damaged and undamaged. Selection of the 318 appropriate number of latent variables for each model was assessed on the basis of the 319 frequency of their occurrence. As shown in Figure 5, this was a clear and 320 unambiguous choice. A summary of the results obtained using mushrooms from day 3210 to day 7 inclusive is shown in Table 3 . In the case of undamaged mushrooms, root mean squared error of cross validation (RMSECV) values achieved were relatively 323 high, only permitting the prediction of post-harvest age of damaged mushrooms to 324 within \pm 2 to 3 days approximately (95\% confidence limit) depending on tissue type. 325 The practical utility of such accuracy levels may be gauged by examination of the 326 SD/RMSECV ratio, all but one of which are below 3.0, the generally accepted 327 minimum value for a model to be of practical utility. With regard to damaged 328 mushrooms, model predictive accuracies were similar for caps and stalks with 329 RMSECV (and RER) values of 1.3 (1.9) and 1.2 (2.) respectively. In the case of gill 330 tissue, better predictive accuracy was achieved with RMSECV and RER values equal 
331 to 0.8 and 3.1 respectively. The number of latent variables associated with these 332 models was low and similar in all cases, with a variation between 6 and 8 only. The 333 application of an objective indicator of the optimum number of PLS loadings to 334 include in any model contributed to their stable performance.

335 The results presented for modeling age in mushrooms using FTIR and 336 chemometrics had misclassification errors of over $30 \%$ (random forests) yielding 337 relatively unsuccessful results. However, random forest models were able to classify 338 day zero samples reasonably well with correct classifications of 82 and $79 \%$ which 339 leads to the possibility of using IR spectroscopy in detecting fresh mushrooms from 340 old mushrooms and could be used within the sector for detecting fraud and 'recycling' 341 of product. The time required for freeze-dried sample preparation is in the order of 342 hours, thus this approach would be applicable for research and quality control 343 purposes.

\section{Acknowledgments:}

347 The authors acknowledge financial support from the Irish Department of Agriculture 348 and Food under the Food Institutional Research Measure (FIRM), supported through 349 EU and national funds. Thanks are due to Dr. Helen Grogan and Ted Cormican, 350 Teagasc, Kinsealy Research Centre, Dublin, Ireland for the supply of mushrooms and 351 background information. 
358 1. Chang, S. T., The world mushroom industry: Trends and technological

359 development. International Journal of Medicinal Mushrooms 2006, 8, 297-317.

3602 2. Teagasc, The Irish Agriculture and Food Development Authority. Teagasc 361 Mushroom Newsletter 2007, 29.

$3623 . \quad$ Brennan, M.; Le Port, G.; Gormley, R., Post-harvest Treatment with Citric 363 Acid or Hydrogen Peroxide to Extend the Shelf Life of Fresh Sliced Mushrooms. 364 Lebensmittel-Wissenschaft und-Technologie 2000, 33, (4), 285-289.

365 4. Burton, K. S.; Noble, R., The influence of flush number, bruising and storage 366 temperature on mushroom quality. Postharvest Biology and Technology 1993, 3, (1), 367 39-47.

368 5. Burton, K. S., Cultural factors affecting mushroom quality - cause and control 369 of bruising. Mushroom Science 2004, 16, 397-402.

370 6. Jolivet, S.; Arpin, N.; Wicher, H. J.; Pellon, G., Agaricus bisporus browning: 371 A review. Mycological Research 1998, 102, 1459-1483.

$3727 . \quad$ Heinemann, P. H.; Hughes, R.; Morrow, C. T.; Sommer, H. J. I.; Beelman, R.; 373 Wuest, P. J., Grading of mushrooms using a machine vision system. Transactions of 374 the American Scoiety of Agricultual Engineers 1994, 37, (5), 1671-1677.

375 8. Harrigan, G. C.; Goodacre, R., Metabolic profiling: its role in biomarker 376 discovery and gene function analysis. Kluwer Academic Publishers: Boston, 2003.

377 9. Melin, A.-M.; Allery, A.; Perromat, A.; Bébéar, C.; Déléris, G.; de Barbeyrac, 378 B., Fourier transform infrared spectroscopy as a new tool for characterization of 379 380 381 382 383 384 mollicutes. Journal of Microbiological Methods 2004, 56, (1), 73-82.

10. Bozkurt, O.; Dincer Bilgin, M.; Severcan, F., The effects of diabetes mellitus on rat skeletal extensor digitorum longus muscle tissue: An FTIR study. Spectroscopy 2007, 21, 151-160.

11. Dogan, A.; Siyakus, G.; Severcan, F., FTIR spectroscopic characterization of irradiated hazelnut (Corylus avellana L.). Food Chemistry 2007, 100, (3), 1106-1114.

12. Toyran, N.; Turan, B.; Severcan, F., Selenium alters the lipid content and protein profile of rat heart: An FTIR microspectroscopic study. Archives of Biochemistry and Biophysics 2007, 458, (2), 184-193.

13. Van de Voort, F. R.; Sedman, J.; Ismail, A. A., A rapid FTIR quality-control method for determining fat and moisture in high-fat products. Food Chemistry 1993, 48, 213-221.

14. Gidman, E.; Goodacre, R.; Emmett, B.; Smith, A. R.; Gwynn-Jones, D., Investigating plant-plant interference by metabolic fingerprinting. Phytochemistry 2003, 63, (6), 705-710.

15. Corte, L.; Rellini, P.; Roscini, L.; Fatichenti, F.; Cardinali, G., Development of a novel, FTIR (Fourier transform infrared spectroscopy) based, yeast bioassay for toxicity testing and stress response study. Analytica Chimica Acta 2010, 659, (1-2), 258-265.

16. Santos, C.; Fraga, M. E.; Kozakiewicz, Z.; Lima, N., Fourier transform infrared as a powerful technique for the identification and characterization of filamentous fungi and yeasts. Research in Microbiology In Press, Corrected Proof.

17. Ammor, M. S.; Argyri, A.; Nychas, G.-J. E., Rapid monitoring of the spoilage of minced beef stored under conventionally and active packaging conditions using Fourier transform infrared spectroscopy in tandem with chemometrics. Meat Science 2009, 81, (3), 507-514. 
18. Rossel, R. A. V.; Behrens, T., Using data mining to model and interpret soil diffuse reflectance spectra. Geoderma In Press, Corrected Proof.

19. Garip, S.; Gozen, A. C.; Severcan, F., Use of Fourier transform infrared spectroscopy for rapid comparative analysis of Bacillus and Micrococcus isolates. Food Chemistry 2009, 113, (4), 1301-1307.

20. Preisner, O.; Lopes, J. A.; Menezes, J. C., Uncertainty assessment in FT-IR spectroscopy based bacteria classification models. Chemometrics and Intelligent Laboratory Systems 2008, 94, (1), 33-42.

21. Kim, H. T.; Kim, S. S.; Kim, S. J. In IEEE Engineering in Medicine and Biology, 27th Annual Conference, Shanghai, China, 2005; Shanghai, China, 2005.

22. Granitto, P. M.; Gasperi, F.; Biasioli, F.; Trainotti, E.; Furlanello, C., Modern data mining tools in descriptive sensory analysis: A case study with a Random forest approach. Food Quality and Preference 2007, 18, (4), 681-689.

23. Zheng, L.; Watson, D. G.; Johnston, B. F.; Clark, R. L.; Edrada-Ebel, R.; Elseheri, W., A chemometric study of chromatograms of tea extracts by correlation optimization warping in conjunction with PCA, support vector machines and random forest data modeling. Analytica Chimica Acta 2009, 642, (1-2), 257-265.

24. Gowen, A. A.; Downey, G.; Esquerre, C.; O'Donnell, C. P., A simple method for estimation of PLS model dimensionality using regression vectors. Journal of Chemometrics 2010, in press.

25. R_Development_Core_Team $R$ : a language and environment for statistical computing, UK, 2007.

26. Belton, P. S.; Colquhoun, I. J.; Grant, A.; Wellner, N.; Field, J. M.; Shewry, P. R.; Tatham, A. S., FTIR and NMR studies on the hydration of a high-Mr subunit of glutenin. International Journal of Biological Macromolecules 1995, 17, (2), 74-80.

27. Di Mario, F.; Rapanà, P.; Tomati, U.; Galli, E., Chitin and chitosan from Basidiomycetes. International Journal of Biological Macromolecules 2008, 43, (1), 812.

28. Workman, J., Methods and Interpretations in "Handbook of Organic Compounds". Academic Press: London, 2001; p 209-242.

29. Mohacek-Grosev, V.; Bozac, R.; Puppels, G. J., Vibrational spectroscopic characterization of wild growing mushrooms and toadstools. Spectrochimica Acta Part A: Molecular and Biomolecular Spectroscopy 2001, 57, (14), 2815-2829.

30. Pierce, J. A.; Rast, D. M., A comparison of native and synthetic mushroom melanins by fourier-transform infrared spectroscopy. Phytochemistry 1995, 39, (1), 49-55.

31. Sandula, J.; Kogan, G.; Kacurakova, M.; Machova, E., Microbial (1->3)-betad-glucans, their preparation, physico-chemical characterization and immunomodulatory activity. Carbohydrate Polymers 1999, 38, 247-253.

32. Pang, H. L.; Lin, A.; Holford, M.; Enerson, B. E.; Lu, B.; Lawton, M. P.; Floyd, E.; Zhao, H., Pathway analysis using random forests classification and regression. Bioinformatics 2006, 16, (22), 2028-2036.

33. Breiman, L., Random Forests. Machine Learning 2001, 45, 5-32.

34. Eastwood, D.; Burton, K., Mushrooms - a matter of choice and spoiling oneself. Microbiology Today 2002, 29, 18-19.

35. Militello, V.; Casarino, C.; Emanuele, A.; Giostra, A.; Pullara, F.; Leone, M., Aggregation kinetics of bovine serum albumin studied by FTIR spectroscopy and light scattering. Biophysical Chemistry 2004, 107, (2), 175-187.

36. Gowen, A. A.; O'Donnell, C.; Taghizadeh, M.; Cullen, P. J.; Frias, J.; Downey, G., Hyperspectral imaging combined with principle component analysis for 
455 bruise damage detection on white mushrooms (Agaricus bisporus). Journal of 456 Chemometrics 2008, 22, 259-267.

457 37. Esquerre, C.; Gowen, A. A.; O'Donnell, C.; Downey, G., Initial studies on the 458 quantitation of bruise damage and freshness in mushrooms using visible-near infrared 459 spectroscopy. Journal of Agricultural and Food Chemistry 2009, 57, 1903-1907.

460 38. Ruiz-Herrera, J., Fungal cell wall, structure, synthesis and assembly. CRC 461 Press: Florida, 1992; p 65. 


\section{FIGURE CAPTIONS}

Figure 1 FTIR transmittance spectra of all mushroom tissues in (a) 400-1800 cm-1 (b) 2800-3050 $\mathrm{cm}-1$, and (c) $3050-4000 \mathrm{~cm}-1$ wavenumber ranges

Figure 2. Average undamaged caps spectrum (raw data)

Figure 3. PC1vs PC2 score plots of undamaged mushroom tissue (a) caps; (b) gills (c) stalks and damaged tissue (d) caps; (e) gills and (f) stalks

Figure 4(a) Relative importance plot of variables that are important in the random forest model for predicting damage/undamaged samples. The variable age being the most important followed by the wavenumbers 1868,1870 and $1845 \mathrm{~cm}^{-1}$. 4(b) Relative importance plot of variables that are important in the random forest model for predicting damaged/undamaged samples when age is not a variable. The most important variables are tissue type followed by the wavenumbers 1868,1870 and $1560 \mathrm{~cm}^{-1}$

Figure 5 Frequency of generation of PLS regression models for mushroom post-harvest age on the basis of the number of latent variables selected. (a) undamaged caps, (b) un damaged gills, (c) undamaged stalks, (d) damaged caps, (e) damaged gills and (f) damaged stalks. Abscissa - no. of latent variables in model; ordinate - number of occurrences

\section{TABLES}

Table.1 Summary of results for mushroom discrimination on the basis of damage

\begin{tabular}{lcccc}
\hline \#Samples & \#Loadings & $\begin{array}{c}\% \\
\text { undamaged } \\
\text { misclassified } \\
\text { mean (std. } \\
\text { deviation) }\end{array}$ & $\begin{array}{c}\text { \% damaged } \\
\text { misclassified } \\
\text { mean (std. } \\
\text { deviation) }\end{array}$ \\
\hline Caps & 160 & 7 & $4.1(4.3)$ & $7.6(4.0)$ \\
Gills & 160 & 9 & $2.1(3.0)$ & $0.8(1.7)$ \\
Stalks & 160 & 12 & $1.7(2.1)$ & $0.6(1.5)$ \\
\hline
\end{tabular}

Table 2 Confusion matrix and the error rate for the prediction of mushroom age. The OOB error rate: $32 \%$. The highlighted numbers are correctly classified samples

\begin{tabular}{lcccccccccc}
\hline & $\mathbf{0}$ & $\mathbf{1}$ & $\mathbf{2}$ & $\mathbf{3}$ & $\mathbf{4}$ & $\mathbf{5}$ & $\mathbf{6}$ & $\mathbf{7}$ & $\begin{array}{c}\text { Error } \\
\text { rate }\end{array}$ \\
\hline $\mathbf{0}$ & $\mathbf{4 9}$ & 3 & 0 & 3 & 2 & 0 & 3 & 2 & 0.18 \\
$\mathbf{1}$ & 1 & $\mathbf{4 2}$ & 2 & 4 & 0 & 1 & 4 & 6 & 0.30 \\
$\mathbf{2}$ & 4 & 5 & $\mathbf{4 3}$ & 2 & 3 & 0 & 0 & 3 & 0.28 \\
$\mathbf{3}$ & 1 & 3 & 5 & $\mathbf{4 7}$ & 2 & 1 & 0 & 1 & 0.22 \\
$\mathbf{4}$ & 3 & 0 & 3 & 3 & $\mathbf{3 2}$ & 2 & 8 & 9 & 0.47 \\
$\mathbf{5}$ & 0 & 0 & 3 & 12 & 3 & $\mathbf{2 9}$ & 4 & 8 & 0.51 \\
$\mathbf{6}$ & 1 & 0 & 6 & 0 & 2 & 0 & $\mathbf{4 8}$ & 3 & 0.20 \\
$\mathbf{7}$ & 2 & 1 & 5 & 2 & 2 & 6 & 8 & $\mathbf{3 4}$ & 0.43 \\
\hline
\end{tabular}

0-7: Sample age in days from day zero to day seven

Error rate: The \% misclassification for each sample age 
Table 3 Summary of PLS regression results for the prediction of post-harvest age (day 0-7 inclusive) in undamaged and damaged mushrooms

\begin{tabular}{cccccc}
\hline Treatment & Tissue & \#Samples & \#Loadings & RMSEC $\boldsymbol{~}^{*}$ & RER $^{* *}$ \\
\hline Undamaged & Caps & 80 & 7 & 1.2 & 2.0 \\
& Gills & 80 & 7 & 1.5 & 1.6 \\
& Stalks & 80 & 7 & 1.2 & 1.9 \\
Damaged & Caps & 80 & 7 & 1.3 & 1.9 \\
& Gills & 80 & 8 & 0.8 & 3.1 \\
& Stalks & 80 & 6 & 1.2 & 2.2 \\
\hline *RMSECV= root mean square error of cross-validation (mean of 100 runs); **RER = SD/RMSECV
\end{tabular}

\section{FIGURES}
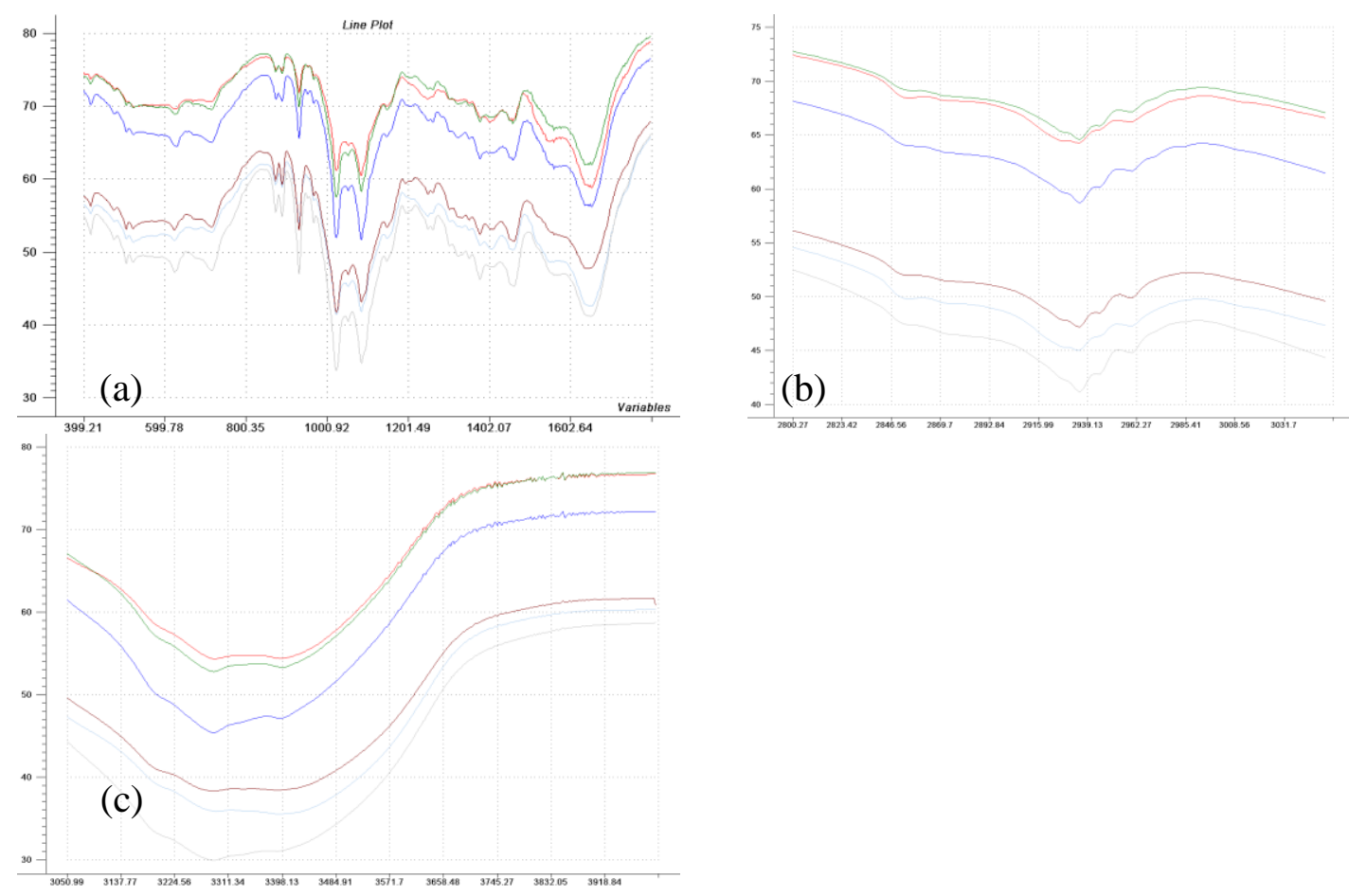

Figure 1 FTIR transmittance spectra of all mushroom tissues in (a) $400-1800 \mathrm{~cm}^{-1}$ (b) $2800-3050$ $\mathrm{cm}^{-1}$, and (c) $3050-4000 \mathrm{~cm}^{-1}$ wavenumber ranges 


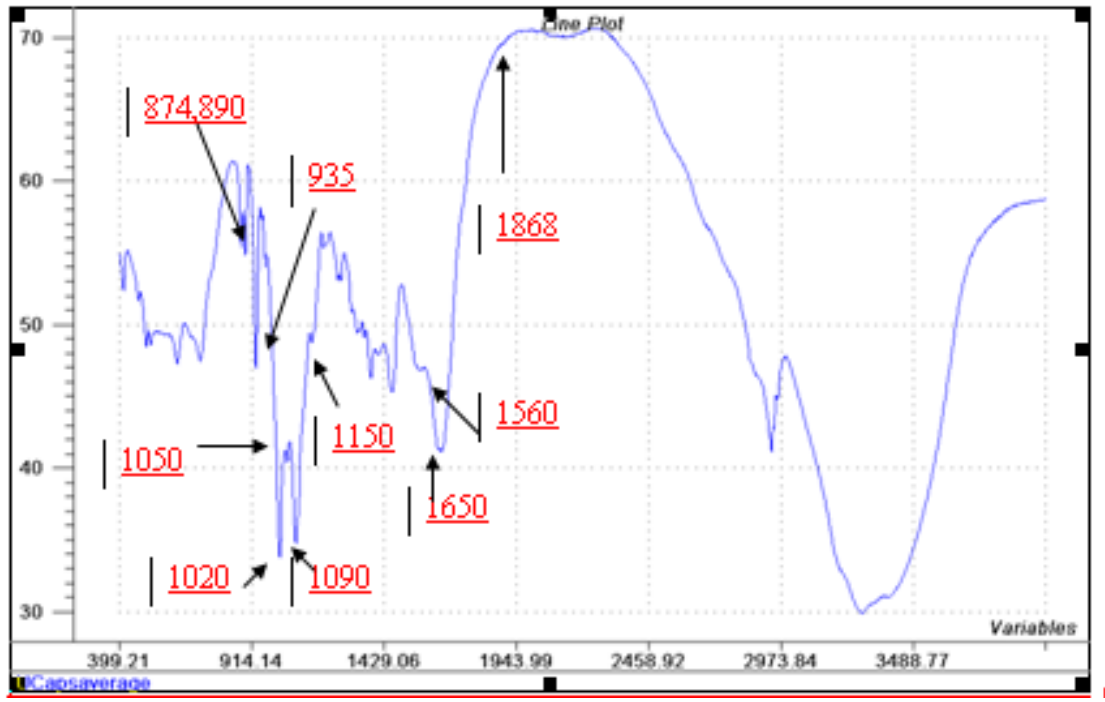

Figure 2. Average undamaged caps spectrum (raw data) 

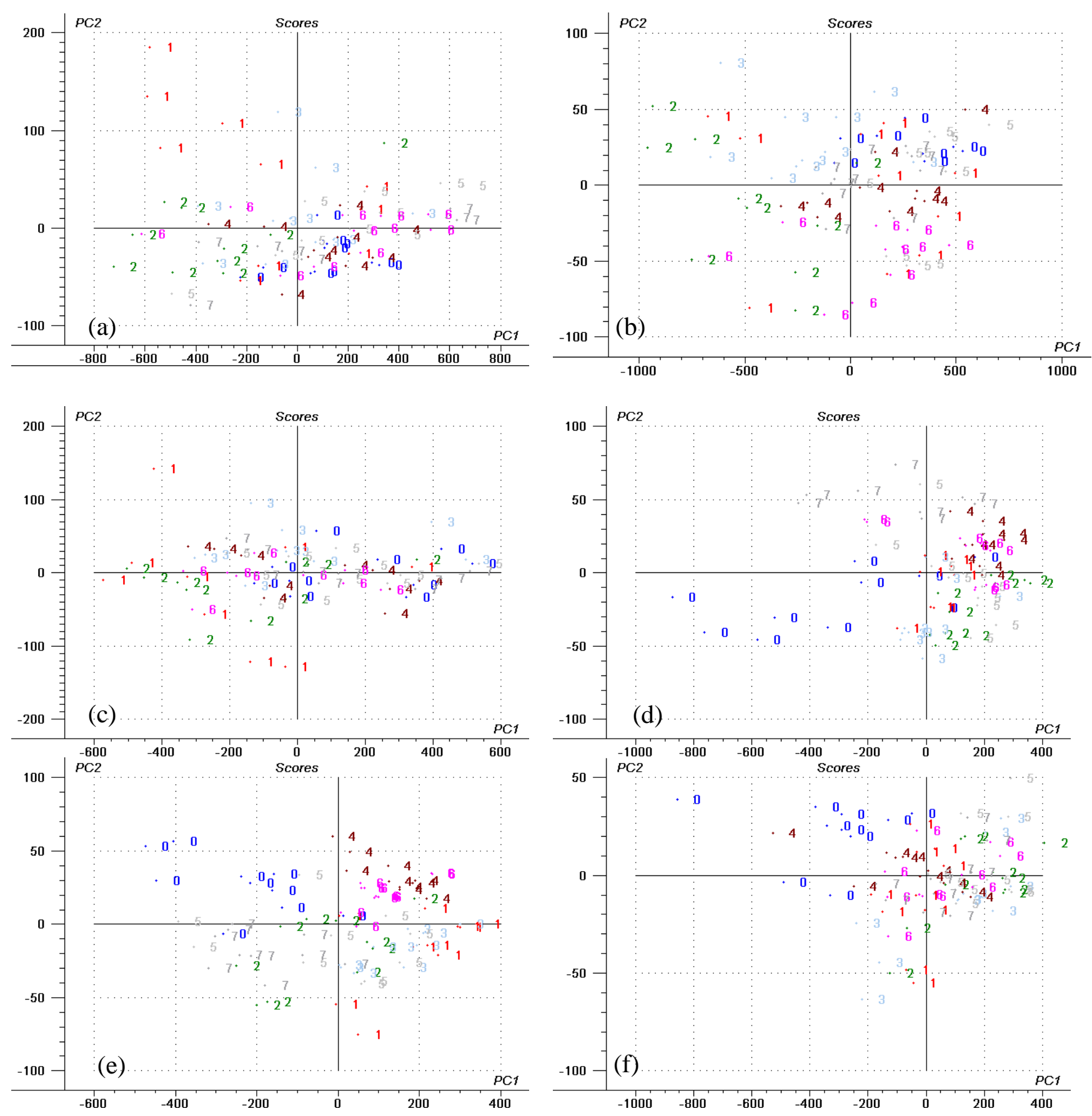

Figure 3 PC1vs PC2 score plots of undamaged mushroom tissue (a) caps; (b) gills (c) stalks and damaged tissue (d) caps; (e) gills and (f) stalks ;0-7: Sample ages from zero to seven 

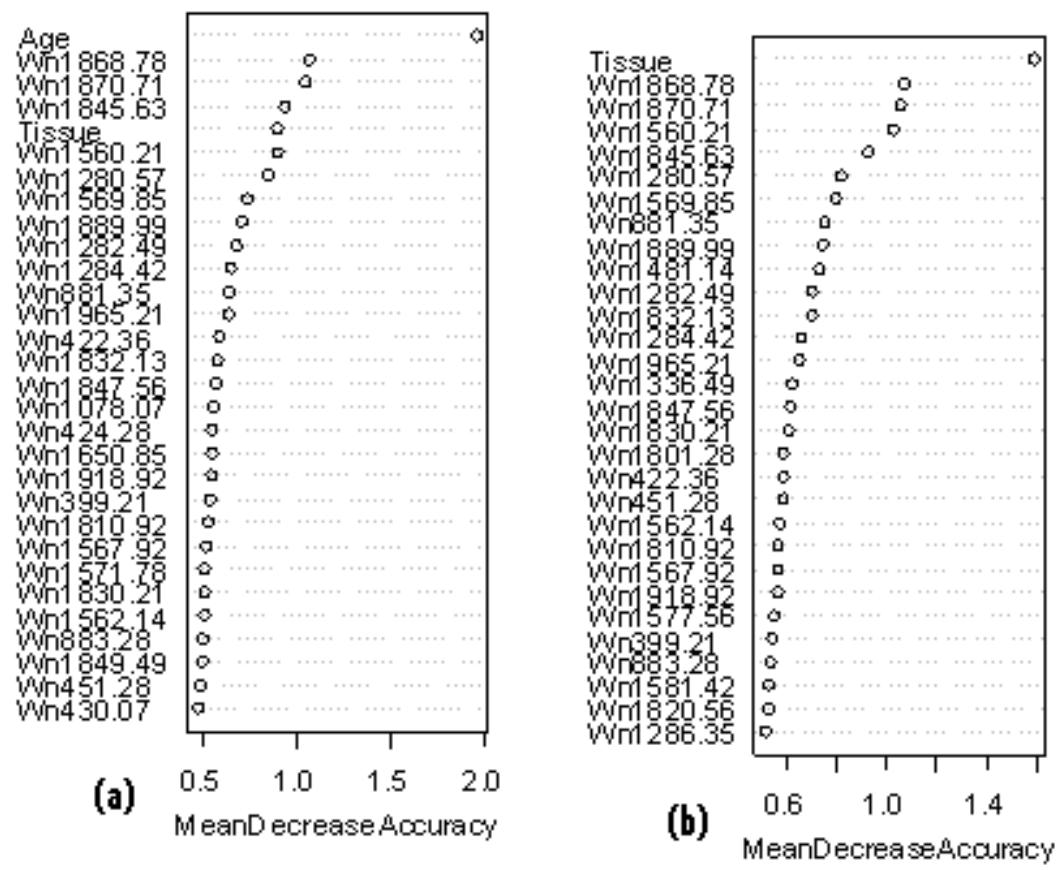

Figure 4(a) Relative importance plot of variables that are important in the random forest model for predicting damage/undamaged samples. The variable age being the most important followed by the wavenumbers 1868,1870 and $1845 \mathrm{~cm}^{-1}$. 4(b) Relative importance plot of variables that are important in the random forest model for predicting damaged/undamaged samples when age is not a variable. The most important variables are tissue type followed by the wavenumbers 1868,1870 and $1560 \mathrm{~cm}^{-1}$ 


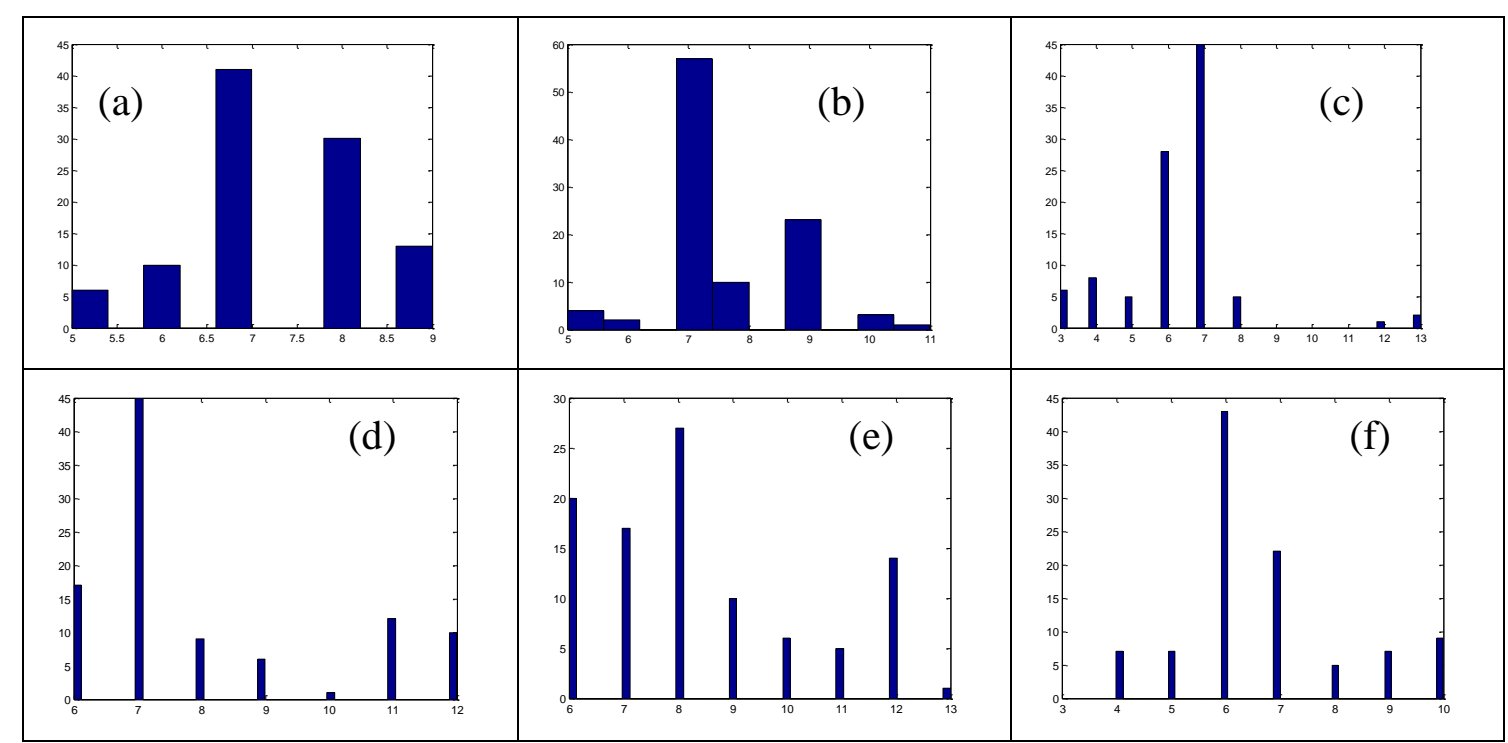

Figure 5. Frequency of generation of PLS regression models for mushroom post-harvest age on the basis of the number of latent variables selected. (a) undamaged caps, (b) un damaged gills, (c) undamaged stalks, (d) damaged caps, (e) damaged gills and (f) damaged stalks. Abscissa - no. of latent variables in model; ordinate - number of occurrences 